\title{
A Bioinspired Neural Model Based Extended Kalman Filter for Robot SLAM
}

\author{
Jianjun Ni, ${ }^{1,2}$ Chu Wang, ${ }^{1}$ Xinnan Fan, ${ }^{1}$ and Simon X. Yang ${ }^{3}$ \\ ${ }^{1}$ College of IOT Engineering, Hohai University, Changzhou 213022, China \\ ${ }^{2}$ Changzhou Key Laboratory of Sensor Networks and Environmental Sensing, Hohai University, Changzhou 213022, China \\ ${ }^{3}$ Advanced Robotics and Intelligent Systems (ARIS) Laboratory, School of Engineering, University of Guelph, \\ Guelph, ON, Canada N1G 2W1
}

Correspondence should be addressed to Jianjun Ni; njjhhuc@gmail.com

Received 15 March 2014; Revised 22 July 2014; Accepted 22 July 2014; Published 13 August 2014

Academic Editor: Yi Chen

Copyright (C) 2014 Jianjun Ni et al. This is an open access article distributed under the Creative Commons Attribution License, which permits unrestricted use, distribution, and reproduction in any medium, provided the original work is properly cited.

\begin{abstract}
Robot simultaneous localization and mapping (SLAM) problem is a very important and challenging issue in the robotic field. The main tasks of SLAM include how to reduce the localization error and the estimated error of the landmarks and improve the robustness and accuracy of the algorithms. The extended Kalman filter (EKF) based method is one of the most popular methods for SLAM. However, the accuracy of the EKF based SLAM algorithm will be reduced when the noise model is inaccurate. To solve this problem, a novel bioinspired neural model based SLAM approach is proposed in this paper. In the proposed approach, an adaptive EKF based SLAM structure is proposed, and a bioinspired neural model is used to adjust the weights of system noise and observation noise adaptively, which can guarantee the stability of the filter and the accuracy of the SLAM algorithm. The proposed approach can deal with the SLAM problem in various situations, for example, the noise is in abnormal conditions. Finally, some simulation experiments are carried out to validate and demonstrate the efficiency of the proposed approach.
\end{abstract}

\section{Introduction}

Robot simultaneous localization and mapping (SLAM) is one of the most challenging problems in mobile robotic fields, which has important theory and application value in various robotic applications, such as the underwater detection, domestic service, and universe exploration [1-5]. Various approaches have been proposed to deal with the SLAM problem. Mullane et al. [6] proposed an integrated Bayesian framework for feature-based SLAM in the general case of uncertain feature number and data association. Lui and Jarvis [7] presented a pure vision-based topological SLAM system for mobile robot autonomous navigation in initially unknown environments. Chatterjee and Matsuno [8] proposed a new neurofuzzy based adaptive Kalman filtering algorithm for SLAM of mobile robots or vehicles. Zhou et al. [9] proposed an auxiliary marginal particle filter and FastSLAM based compositive SLAM algorithm to improve the performance of samples and increase the estimation accuracy. Kaess et al. [10] presented a novel approach to the simultaneous localization and mapping problem that is based on fast incremental matrix factorization. Benedettelli et al. [11] proposed a multirobot cooperative SLAM algorithm using M-Space representation of linear features, suitable for environments which can be represented in terms of lines and segments.

Previous research on SLAM problem may be divided into two broad categories. One category consists of a number of mathematical probabilistic techniques, such as EKF based algorithm [12, 13], particle filtering based algorithm [14, 15], and Monte Carlo localization method [16, 17]. The other category of research focuses on emulating the biological systems thought to be responsible for mapping and navigation in animals [18-20]. However, both of the category algorithms have some limitations. For example, the standard Kalman filtering is based on the minimum variance estimation; the filter will exhibit a divergence phenomenon with the increase in the number of filterings; namely, the error between the estimated value and the actual value will become bigger and bigger. The biological emulating based algorithms are too 
complex to be realized and the SLAM mechanism of animals is not very clear now.

Although there are many modern techniques that are suitable for SLAM, the EKF based SLAM method is still one of the most popular methods for SLAM, because the EKF based method has a good algorithm structure for SLAM and a strict mathematical theory basis. The EKF based method is used widely to solve SLAM problem [8, 21-24], especially in some applications, where the computational constraints or the scarcity of high precision sensors makes it impossible to use other SLAM methods, such as the methods based on highresolution vision sensors [25-27] and the methods based on high dense topological maps [28, 29]. However, there is a key problem of the EKF based SLAM method; namely, the accuracy of the system noise and the observation noise model will decide the final accuracy of the SLAM algorithm. To deal with this problem, some improved algorithms have been proposed. For example, Du et al. [30] proposed an improved fuzzy adaptive EKF to establish a priori noise model for the SLAM problem. Kang et al. [31] presented a modified neural network aided EKF based SLAM for improving the accuracy of the feature map. However, those algorithms introduced above still have some limitations, such as the computation which is complex and the algorithm is unstable in some conditions.

To improve the robustness and accuracy of the EKF based SLAM algorithm, a novel adaptive EKF based SLAM approach is proposed in this paper. Firstly, an adaptive EKF based SLAM algorithm structure is proposed, where the weights of noises in the EKF based SLAM algorithm are adjusted adaptively. Secondly, a bioinspired neural model is used to realize these adjustments for noise weights, which can guarantee the stability of the filter. Finally, some simulation experiments were conducted. The results show the efficiency of the proposed approach. The divergence problem of EKF is solved effectively, and the robustness and accuracy of the SLAM approach are improved.

This paper is organized as follows. Section 2 presents the proposed bioinspired neural model based extended Kalman filter algorithm. The simulation experiments for mobile robot SLAM task are given in Section 3. Section 4 discusses the sensitivity of the parameters and some performances of the proposed approach in detail. Finally, the conclusions are given in Section 5.

\section{The Proposed Bioinspired Neural Model Based EKF Algorithm}

In this paper, the SLAM problem is studied. The core task of SLAM is that a robot explores in an unknown environment to learn the environment (map), while simultaneously using that map to locate within the environment [32-34]. A novel approach based on bioinspired neural model is proposed. In the proposed approach, EKF is used to solve the SLAM problem, while a bioinspired neural model is used to adjust the weights of system noise and observation noise adaptively in the EKF based SLAM algorithm. The main reason to use this bioinspired neural model is that it is not only a feasible solution, but also an efficient one. The bioinspired method can deal with SLAM problem efficiently without any a priori knowledge nor any learning procedures, which is a trend in the SLAM field $[32,35]$. The basic work flow and the theoretical analysis of the EKF based SLAM algorithm can be viewed in $[8,36,37]$. The proposed approach is introduced in detail as follows.

2.1. The SLAM Algorithm Based on EKF. The basic idea of EKF based SLAM is that the robot uses the known states to predict the states of the next step, and then this prediction is corrected based on the observation at the next step. The state equation of EKF based algorithm is as follows [38]:

$$
\begin{aligned}
X(k+1) & =F(k) X(k)+G(k) U(k)+W(k), \\
Z(k) & =H(k) X(k)+V(k),
\end{aligned}
$$

where $X(k)$ is the state variable of the system at time $k ; U(k)$ is the control variable of the system; $Z(k)$ is the observation value by robotic sensors; $F(k)$ is the state transition matrix; $G(k)$ is the control matrix; $H(k)$ is the observation matrix; $W(k)$ and $V(k)$ are the noise of system and observation, respectively. Here the state of the system is based on the specific SLAM tasks, such as the number of landmarks and the dimension of the environment. Assume that the location of the robot at time $k$ is $x_{v}(k)$ and the position of the $i$ th landmark is $l_{i}$; then the state of the system can be denoted by

$$
X(k)=\left[x_{v}^{T}(k), l_{1}^{T}, l_{2}^{T}, \ldots, l_{N}^{T}\right]^{T},
$$

where $N$ is the number of landmarks in the environment. The location of the robot $x_{v}(k)$ is updated by its kinematic model, while the landmarks in the environment are assumed as static, namely, $l_{i}(k+1)=l_{i}(k)=l_{i}$. The general EKF based SLAM algorithm includes two main steps.

(1) Prediction Step. Firstly, the state equation is used to predict the state of system at the next time. Assume that the system state calculated at time $k$ is $X(k \mid k)$; the prediction of system state for the next time $k+1$ is denoted by $X(k+1 \mid k)$, which can be predicted as follows:

$$
\begin{aligned}
& X(k+1 \mid k)=F(k) X(k \mid k)+G(k) U(k), \\
& Z(k+1 \mid k)=H(k) X(k+1 \mid k), \\
& P(k+1 \mid k)=F(k) P(k \mid k) F^{T}(k)+Q(k),
\end{aligned}
$$

where $P(k+1 \mid k)$ and $P(k \mid k)$ are the covariance matrix related to $X(k+1 \mid k)$ and $X(k \mid k)$, respectively; and $Q(k)$ is the covariance matrix of control noise.

(2) Update Step. When the observation of system at time $k+1$ is obtained by the onboard sensors of the robot, the system state at time $k+1$ can be updated based on the system state equation and the prediction of system state:

$$
\begin{aligned}
& X(k+1 \mid k+1) \\
& \quad=X(k+1 \mid k)+K(k+1)(Z(k+1)-Z(k+1 \mid k)), \\
& P(k+1 \mid k+1) \\
& \quad=P(k+1 \mid k)-K(k+1) H(k+1) P(k+1 \mid k),
\end{aligned}
$$


where $K(k)$ is the gain of EKF, which can be obtained by

$$
K(k+1)=\frac{P(k+1 \mid k) H^{T}(k+1)}{H(k+1) P(k+1 \mid k) H^{T}(k+1)+R(k+1)},
$$

where $R(k)$ is the covariance matrix of observation noise.

In this paper, the difference between the prediction of system state and the real value obtained by the robotic onboard sensors is defined as the innovation, which is denoted by $C(k)$. The innovation $C(k)$ can be calculated by

$$
C(k+1)=Z(k+1)-Z(k+1 \mid k) .
$$

The variance of the innovation $C(k)$ is defined as $S(k)$, which can be calculated by

$$
S(k+1)=H(k+1) P(k+1 \mid k) H^{T}(k+1)+R(k+1) .
$$

The innovation $C(k)$ can be used to adjust and correct the EKF based method. In this paper, an adaptive EKF based SLAM algorithm is proposed, where a bioinspired neural model is used to adjust the credibility and availability of the observation.

2.2. The Proposed Adaptive Model for the EKF Based SLAM. Before the introduction of the bioinspired neural model based EKF algorithm, it is necessary to set up a control model for the EKF based SLAM firstly [30]. In this model, the noises in the EKF based SLAM algorithm are expressed as follows:

$$
\begin{aligned}
& Q(k)=a(k) Q, \\
& R(k)=b(k) R,
\end{aligned}
$$

where $Q$ and $R$ are the initial covariance matrixes for noises; $a(k)$ and $b(k)$ are the weights for system noise and observation noise, respectively.

In a certain time window $M$, the mean values of the innovation $C(k)$ and its variance $S(k)$ are denoted by $\bar{C}(k)$ and $\bar{S}(k)$, respectively, which can be calculated as follows:

$$
\begin{aligned}
& \bar{C}(k)=\frac{1}{M} \sum_{i=k-M+1}^{k} C(i), \\
& \bar{S}(k)=\frac{1}{M} \sum_{i=k-M+1}^{k} S(i) S^{T}(i) .
\end{aligned}
$$

The confidence levels of the mean values of the innovation $C(k)$ and its variance $S(k)$ are denoted by $q_{1}$ and $q_{2}$, respectively, which can be obtained by

$$
\begin{aligned}
& q_{1}(k)=\frac{(1 / J) \sum_{j=1}^{J} \bar{C}_{j}(k)}{Z_{j}(k)} \\
& q_{2}(k)=\frac{\operatorname{Tr}(\bar{S}(k))}{\operatorname{Tr}(S(k))},
\end{aligned}
$$

where $J$ is the dimension of the observation and the $\operatorname{Tr}()$ is a function to calculate the trace of a matrix. In the EKF model, $q_{1}$ and $q_{2}$ will fluctuate around 0 and 1 in a very little range, respectively, if the assumption of the noise model is accurate. Otherwise, the values of $\bar{C}(k)$ and $\bar{S}(k)$ will increase when the real value of the observation noise increases. Then the value of $q_{1}$ will be far away from 0 and the value of $q_{2}$ will be bigger than 1 . Under this condition, the stability of the system will become worse $[30,36,38]$.

Remark 1. Of course, if the parameters of the noise model are properly tuned and assuming correspondences are known, the covariance of the noise will become arbitrarily small and the accuracy of the EKF based SLAM algorithm can be guaranteed. But it needs more experience and time, which are both scarce in the real-world applications of SLAM tasks.

To deal with the problem discussed above, an adaptive model is proposed in this paper. The basic idea of this model is to change the weights of the noises if the assumption of the noise model is away from the actual value. The adaptive EKF based SLAM algorithm structure is shown in Figure 1. In this paper, a bioinspired neural model is used to realize the adjustment function.

2.3. A Bioinspired Neural Model for the EKF Based SLAM. In the proposed approach, a bioinspired neural dynamic model is used to adjust the weights of noises adaptively. The first computational model of a uniform patch of membrane in a biological neural system using electrical circuit elements was proposed by Hodgkin and Huxley [39, 40]. In their membrane model, the dynamics of the voltage across the membrane is described using a state equation technique (see $[39,41])$. Based on this state equation, a shunting model is obtained as

$$
\frac{d x_{i}}{d t}=-A x_{i}+\left(B-x_{i}\right) S_{i}^{+}-\left(D+x_{i}\right) S_{i}^{-},
$$

where $x_{i}$ is the neural activity (membrane potential) of the $i$ th neuron; $A, B$, and $D$ are nonnegative constants, representing the passive decay rate, and the upper and lower bounds of the neural activity, respectively; and $S_{i}^{+}$and $S_{i}^{-}$are the excitatory and inhibitory inputs to the neuron. This shunting model was first proposed by Grossberg to understand the real-time adaptive behavior of individuals to complex and dynamic environmental contingencies and has many applications in visual perception, sensory motor control, and many other areas (e.g., [41, 42]). This shunting model is stable [39, 43], where the neural activity $x_{i}$ is bounded in the finite interval $[-D, B]$. The state workspace varies in terms of the dynamics of the neural model, due to the influence of the external inputs.

In the proposed EKF based SLAM algorithm structure (see Figure 1), the prediction and update steps are carried out continuously. As a result, the confidence levels of the mean values of the innovation $C(k)$ and its variance $S(k)$ will change constantly. Because the system tends to be stable if $q_{1}$ is close to 0 and $q_{2}$ is close to 1 , the weights $a(k)$ and $b(k)$ of the system 


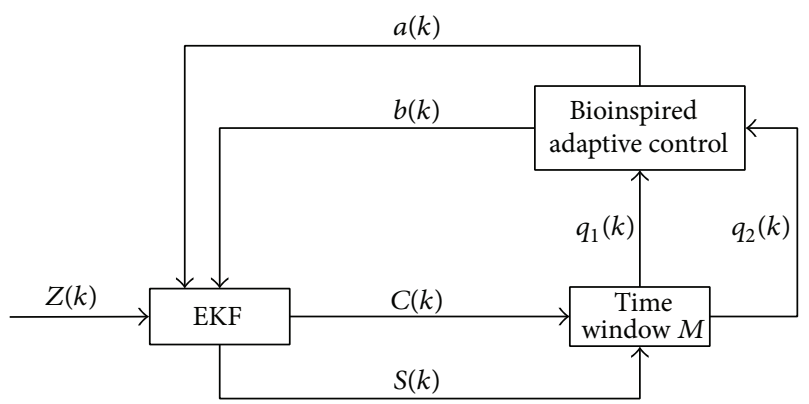

FIGURE 1: The adaptive EKF based SLAM algorithm structure based on the bioinspired neural model.

noise and observation noise in the EKF based SLAM algorithm should be controlled in real time. As introduced above, the bioinspired neural model has several advantages such as the guaranteed stability and efficient computation [41,44], so it could be used to improve the adaptability of the EKF based SLAM algorithm. The basic idea of the proposed approach is to realize the control function based on the bioinspired neural model above. The inputs of this controller are $q_{1}(k)$ and $q_{2}(k)$, and the outputs of this controller are $a(k)$ and $b(k)$. The adaptive control function based on this bioinspired neural model (namely, the shunting model above; see (11)) is defined as

$$
\begin{aligned}
& \frac{d a(k)}{d t}=-A_{1} a(k)+\left(B_{1}-a(k)\right) f_{1}-\left(D_{1}+a(k)\right) f_{2}, \\
& \frac{d b(k)}{d t}=-A_{2} b(k)+\left(B_{2}-b(k)\right) f_{1}-\left(D_{2}+b(k)\right) f_{2},
\end{aligned}
$$

where $A_{1}, A_{2}, B_{1}, B_{2}, D_{1}$, and $D_{2}$ are the parameters of the controller for $a(k)$ and $b(k) ; f_{1}$ and $f_{2}$ are the inputs of the bioinspired neural model, which are defined as

$$
\begin{aligned}
& f_{1}=q_{1}(k), \\
& f_{2}=1-q_{2}(k) .
\end{aligned}
$$

Remark 2. Based on the proposed bioinspired model, the weights for system noise and observation noise (namely, $a(k)$ and $b(k))$ can be guaranteed in a stable range and change with the difference between the prediction of the system state and the real value obtained by the robotic onboard sensors (namely, $q_{1}(k)$ and $q_{2}(k)$ ) adaptively. In addition, this bioinspired neural model based controller does not need any a priori knowledge about the noise model nor any learning procedures.

\section{Simulation Experiments}

In this paper, to test the performance of the proposed approach, some simulation experiments are conducted which were coded in MATLAB. In these experiments, a robot with some sensors moves in a predetermined trajectory and some SLAM algorithms are used to map the environment and keep track of the robot position simultaneously (see Figure 2). In these experiments, the noise model is unknown.

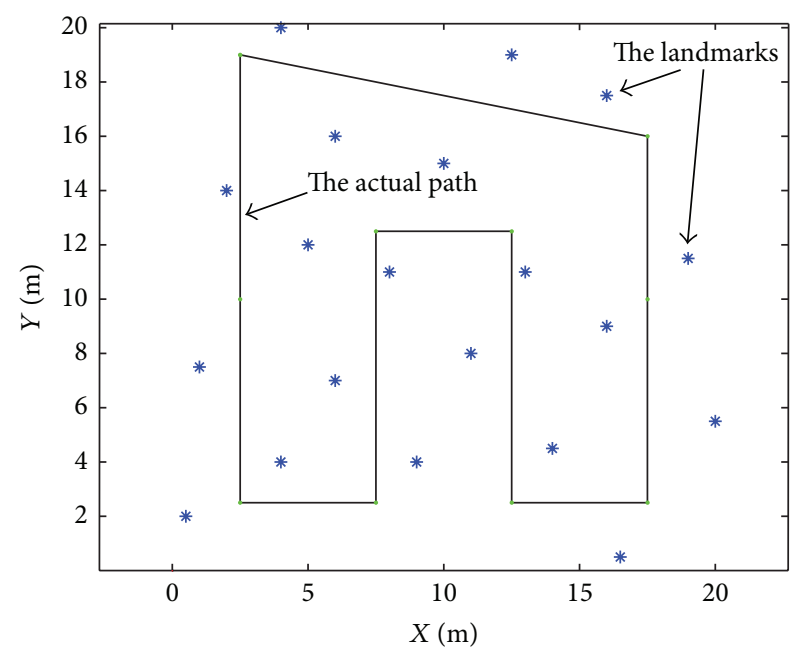

FIGURE 2: The simulation environment of the SLAM task.

TABLE 1: The values of parameters in the experiments.

\begin{tabular}{lcc}
\hline Parameters & Value & Remarks \\
\hline$A_{1}$ and $A_{2}$ & 1 & $\begin{array}{c}\text { The passive decay rate of the } \\
\text { inputs to EKF }\end{array}$ \\
$B_{1}$ and $B_{2}$ & 1.5 & The upper bounds of the inputs \\
to EKF \\
$D_{1}$ and $D_{2}$ & -1.5 & The lower bounds of the inputs \\
to EKF
\end{tabular}

For simplification without loss of generality, the noise is given out artificially in the simulation experiments. The specific type of sensors and the noise function for a given sensor are ignored in this paper. To show the advantages of the proposed bioinspired neural model based EKF approach (B-EKF), it is compared with the standard EKF algorithm (S-EKF) and the fuzzy logic based EKF algorithm (F-EKF). To remove the random effects on the SLAM algorithms, every experiment was conducted 15 times (about 5000 steps). The parameters in all of the experiments are the same, which are listed in Table 1.

3.1. The Experiment under Normal Noise Condition. In this experiment, the noise of the observation is under normal condition; namely, the noise will fluctuate in a little range during the whole moving process of the robot (see Figure 3). The results of the SLAM based on the proposed approach in this experiment are shown in Figure 4. Figure 5 is the localization error of the robot and the estimated error of the landmarks based on the S-EKF method, F-EKF method, and B-EKF method, respectively. The comparisons of the localization errors in the SLAM task are listed in Table 2.

Remark 3. The root mean squared error (RMSE) in Table 2 is calculated by

$$
\mathrm{RMSE}=\sqrt{\frac{1}{n} \sum_{i=1}^{n}\left(e_{i}-\widehat{e}_{i}\right)^{2}},
$$




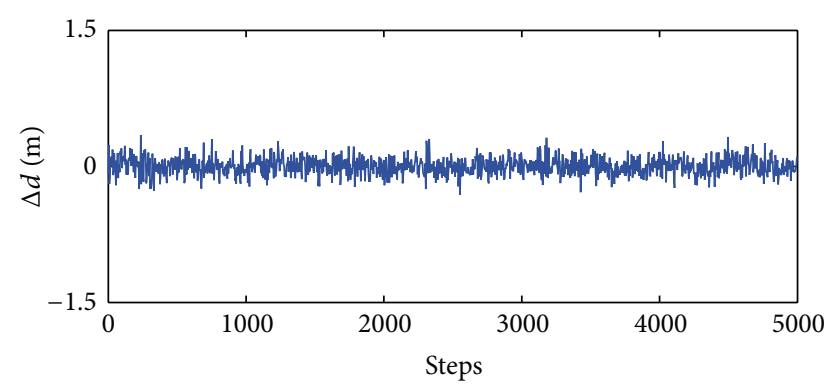

(a)

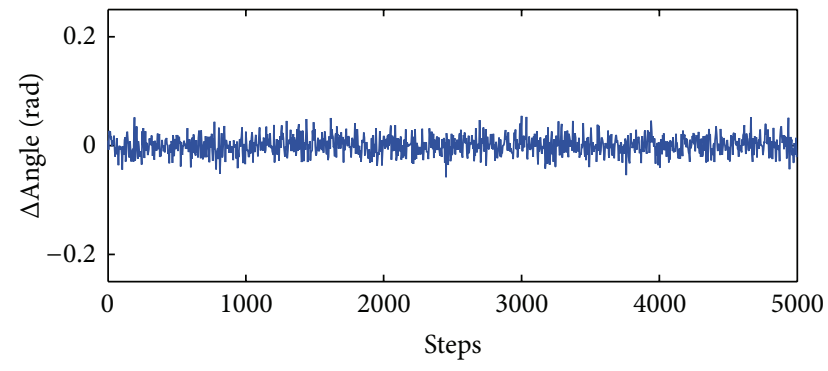

(b)

FIGURE 3: The noise distribution designed for the first experiment: (a) the error of the distance observation; (b) the error of the angle observation.

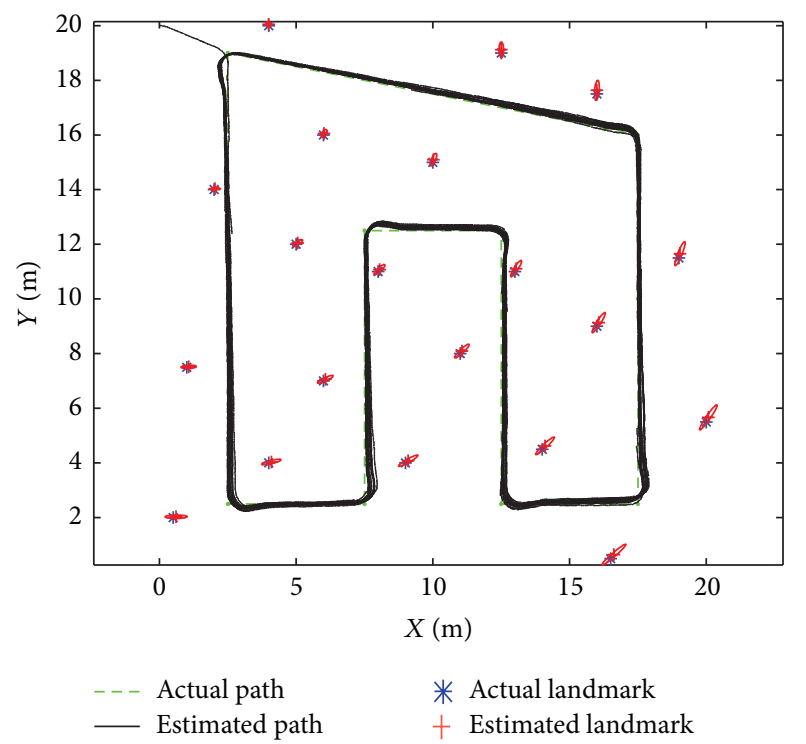

FIGURE 4: The results of the SLAM task based on the proposed approach in the first experiment.

where $e_{i}$ and $\widehat{e}_{i}$ are the real value and the predicted value of the robot position at the $i$ th step, respectively.

The results in Figure 4 show that the trajectory calculated by B-EKF is closest to the actual trajectory of the robot, and the estimated landmarks are closest to the actual landmarks. The results of the localization error and the estimated error of the landmarks in Figure 5 show that all the three methods can complete the SLAM task under the normal noise condition.
TABLE 2: The root mean square error of the SLAM task in the first experiment.

\begin{tabular}{lccc}
\hline \multirow{2}{*}{$\begin{array}{l}\text { The algorithms } \\
\text { for the SLAM task }\end{array}$} & \multicolumn{3}{c}{ The root mean square error } \\
& $\Delta X / \mathrm{m}$ & $\Delta Y / \mathrm{m}$ & $\Delta$ Angle/rad \\
\hline The S-EKF based method & 0.2707 & 0.2366 & 0.0829 \\
The F-EKF based method & 0.1205 & 0.1473 & 0.0472 \\
The B-EKF based method & 0.0976 & 0.0816 & 0.0105 \\
\hline
\end{tabular}

TABLE 3: The root mean square error of the SLAM task in the second experiment.

\begin{tabular}{lccc}
\hline The algorithms & \multicolumn{3}{c}{ The root mean square error } \\
for the SLAM task & $\Delta X / \mathrm{m}$ & $\Delta Y / \mathrm{m}$ & $\Delta$ Angle/rad \\
\hline The S-EKF based method & 0.4064 & 0.3922 & 0.0921 \\
The F-EKF based method & 0.3083 & 0.3316 & 0.0496 \\
The B-EKF based method & 0.1132 & 0.0918 & 0.0204 \\
\hline
\end{tabular}

However, the error of the proposed approach is less than both the F-EKF and S-EKF methods (see Table 2) and the fluctuation of errors in the proposed approach is very little, which shows that the proposed approach can deal with the SLAM problem stably (see Figure 5). The results in this experiment show that the proposed approach has a good performance to deal with the SLAM problem under normal noise condition.

3.2. The Experiment under Abnormal Noise Condition. To further test the performance of the proposed approach, this experiment is conducted, where the noise will fluctuate with the time violently (see Figure 6), which would happen if the performance of the onboard sensors becomes bad (such as the temperature effects). The results of the SLAM task based on the proposed approach are shown in Figure 7. Figure 8 is the localization error of the robot and the estimated error of the landmarks in this experiment. The comparisons of the localization errors in the SLAM task are listed in Table 3.

In this experiment, with the increase of noises, it is hard for the S-EKF method to adapt to this change; the reliability of the observations reduces and the filter becomes unstable, so the localization error and the estimated error will increase and fluctuate violently (see Figure 8(a)). In the F-EKF method, the error will increase obviously too (see Figure 8(b)), although it is less than the S-EKF method. The main reason is that the fuzzy rule in the F-EKF method is fixed, which cannot deal with this abnormal noise condition very well. However, in the proposed approach, with the increasing of noises, the bioinspired adaptive controller of the B-EKF method adjusts the weights of system noise and observation noise in real time, and the positioning accuracy will be significantly improved. During the whole exploration process of the robot, the localization error and the estimated error always fluctuate in a small range (see Figure 8(c)). The results in Figure 8 and Table 3 show that the proposed approach can deal with this abnormal noise condition in the SLAM task efficiently. 

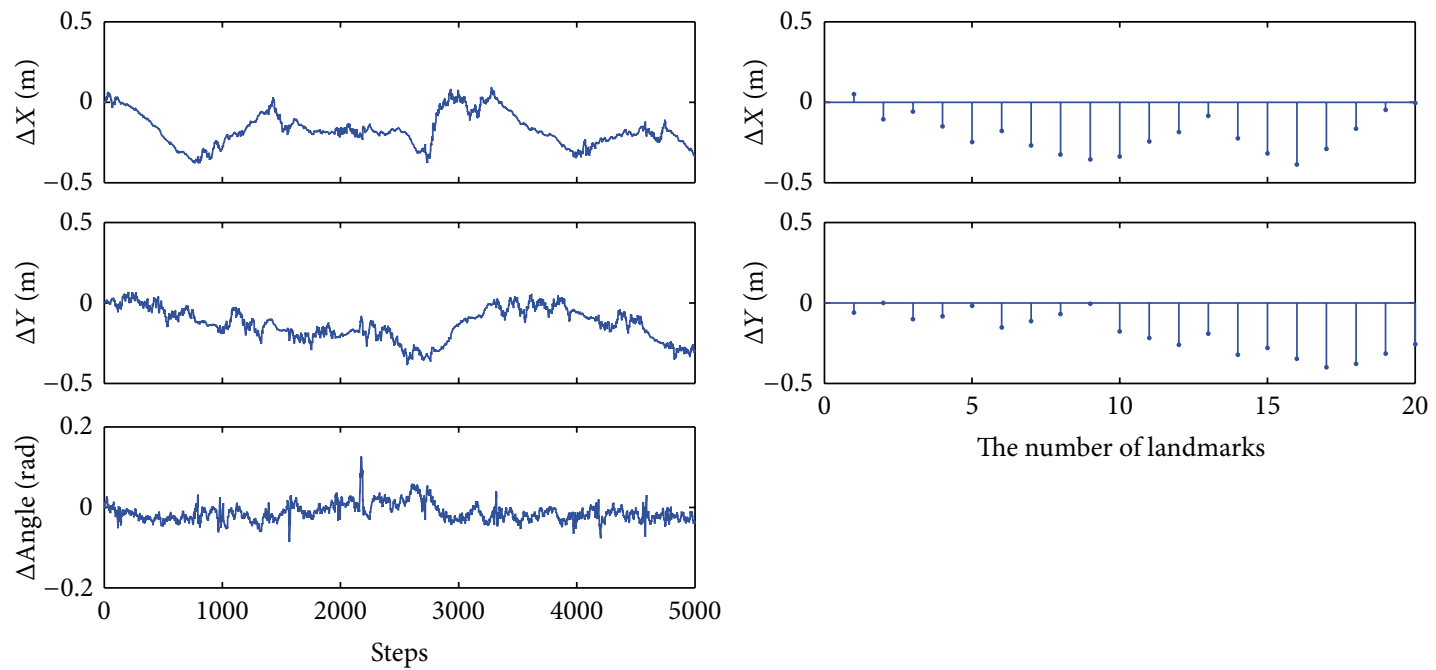

(a)
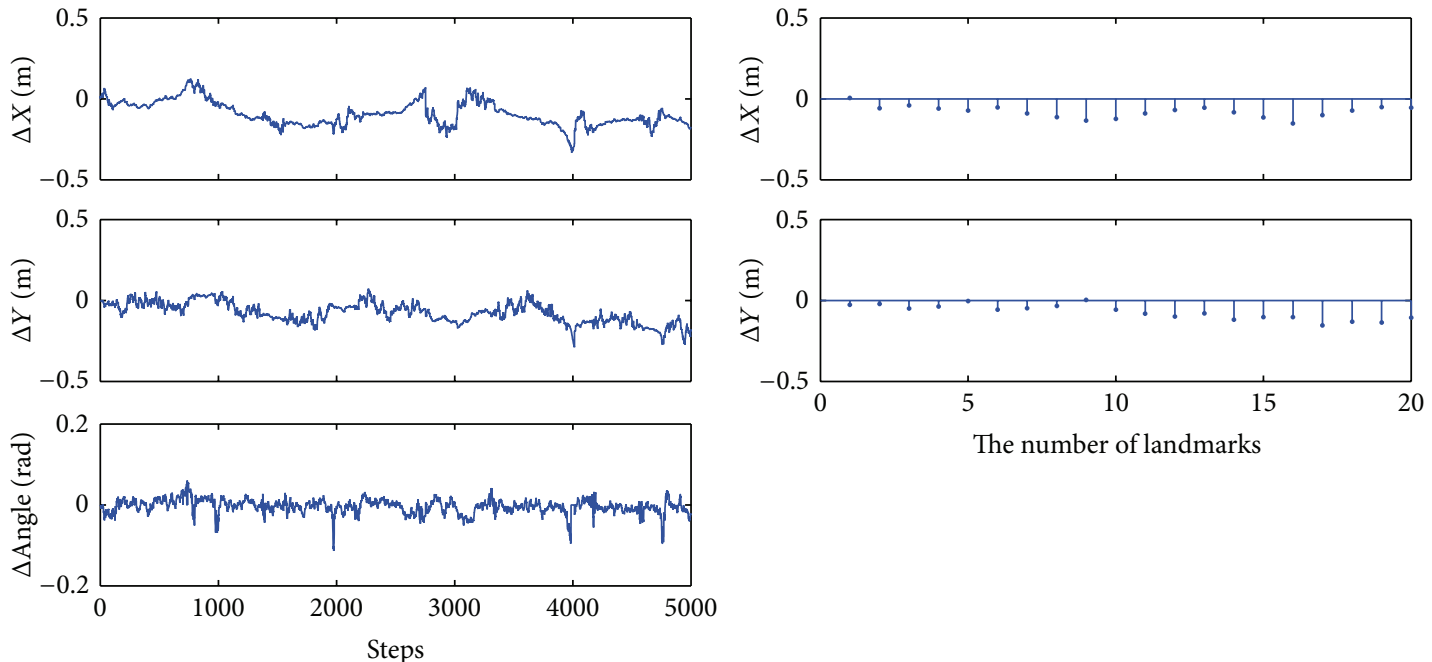

(b)
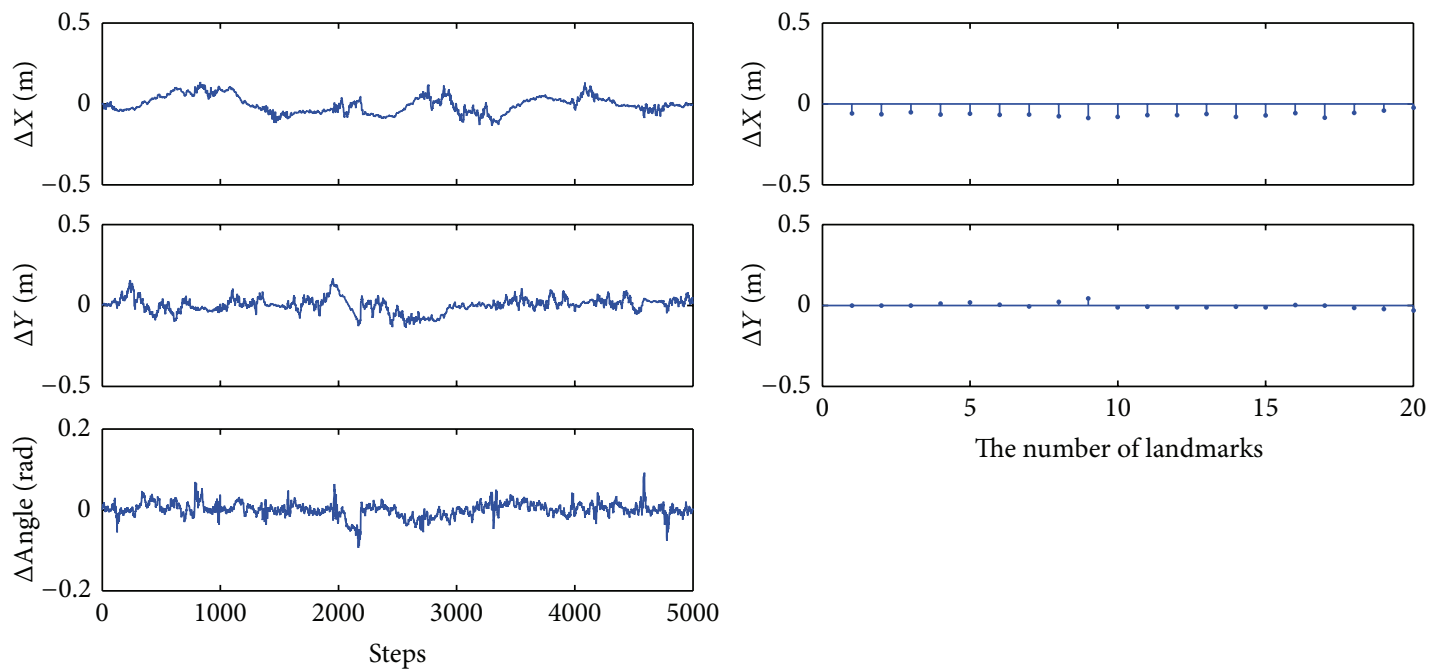

(c)

FIGURE 5: The localization error and the estimated error in the first experiment: (a) based on S-EKF method; (b) based on F-EKF method; (c) based on B-EKF method. 


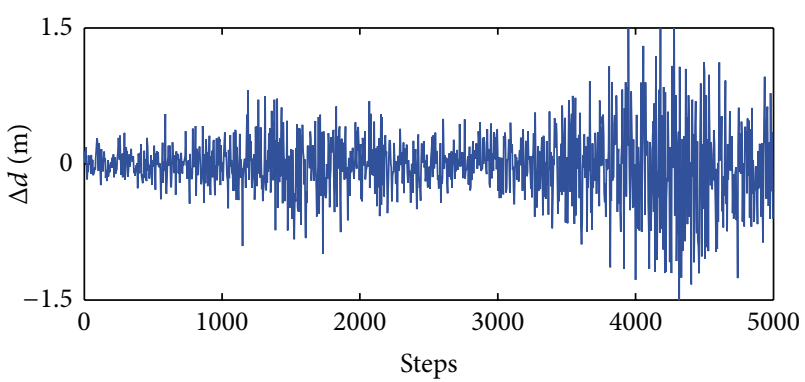

(a)

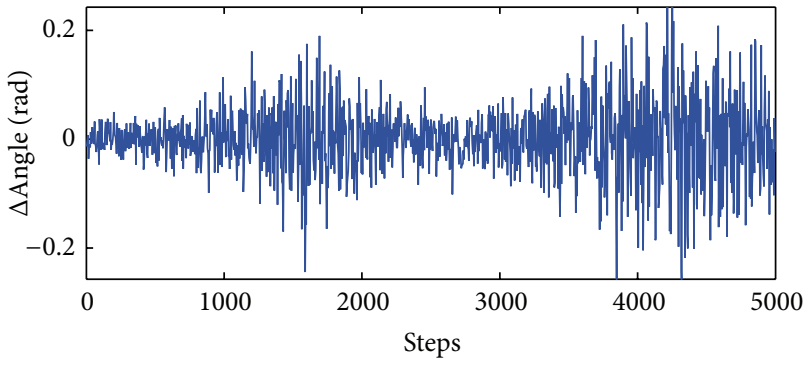

(b)

Figure 6: The noise distribution designed for the second experiment: (a) the error of the distance observation; (b) the error of the angle observation.

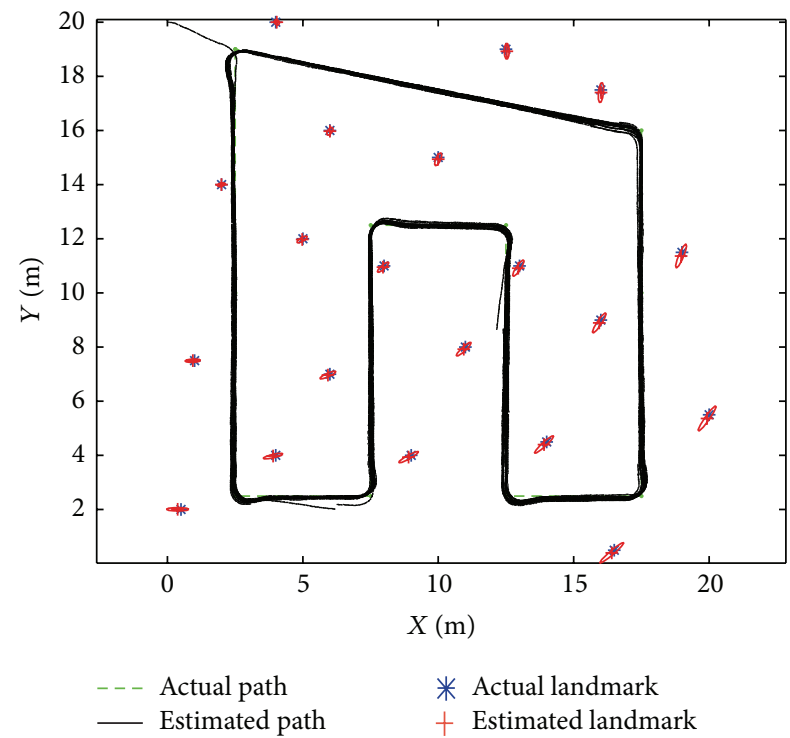

Figure 7: The results of the SLAM task based on the proposed approach in the second experiment.

\section{Discussions}

The results of the simulation experiments in Section 3 show that the proposed approach can satisfy the SLAM task under various situations. The parameter sensitivity and some performances of the proposed approach are discussed in this section.
TABLE 4: The root mean square error of the SLAM task by different parameters $A_{1}$ and $A_{2}$.

\begin{tabular}{lccc}
\hline The value of the parameters & \multicolumn{3}{c}{ The root mean square error } \\
$A_{1}$ and $A_{2}$ & $\Delta X / \mathrm{m}$ & $\Delta Y / \mathrm{m}$ & $\Delta$ Angle/rad \\
\hline 0.5 & 0.1176 & 0.0982 & 0.0218 \\
1 & 0.1132 & 0.0918 & 0.0204 \\
1.5 & 0.1148 & 0.0942 & 0.0270 \\
2 & 0.1262 & 0.1114 & 0.0385 \\
\hline
\end{tabular}

At first, the parameters of the proposed approach are discussed. There are many discussions on the parameters of the bioinspired neural model in our previous work [41, $43,45]$. The upper and lower activity bounds $B$ and $D$ in the proposed bioinspired neural model will only effect the relative range of the weights $a(k)$ and $b(k)$ for the system noise and observation noise in the EKF based SLAM algorithm (see (8) and (12)), which can be decided by the real noise range in the SLAM tasks. So here just the parameters $A_{1}$ and $A_{2}$ are discussed, which are very important in the bioinspired neural model. To analyze the influence of parameters $A_{1}$ and $A_{2}$, some simulations were carried out under the same conditions as the second experiment in Section 3. The results are listed in Table 4. The results in Table 4 show that the proposed approach is not very sensitive to the variations of the parameters $A_{1}$ and $A_{2}$ even in the abnormal noise conditions. So the parameters can be chosen in a very wide range. All the cases studied in this paper use the same parameters, which are listed in Table 1.

To discuss the adaptability of the proposed approach in different SLAM tasks, two simulation experiments were conducted, where the noise distribution and the parameters of the proposed approach are the same as the second experiment in Section 3, except that the trajectory of the robot and the distribution of the landmarks are different. The results of these experiments based on the proposed approach are shown in Figure 9. The root mean square errors of the proposed approach in these SLAM tasks with the simple trajectory and the complex trajectory are $\{\Delta X / \mathrm{m}=0.1020, \Delta Y / \mathrm{m}=0.0921$, $\Delta$ Angle $/ \mathrm{rad}=0.0217\}$ and $\{\Delta X / \mathrm{m}=0.1103, \Delta Y / \mathrm{m}=0.0928$, $\Delta$ Angle/rad $=0.0209\}$, respectively. The errors in the two experiments are both very little, which show that the proposed approach can deal with various SLAM tasks efficiently.

The process introduced in Section 2 and the simulation results of the SLAM tasks show that the proposed approach has some good performances. For example, the adaptivity of the proposed approach is good, which can adjust the filter in real time based on the noise conditions. Furthermore, the proposed approach can extend to real-world SLAM applications, and nothing needs to be done on the algorithm. The proposed approach is different from other algorithms, which need a priori knowledge or learning process [30, 46].

\section{Conclusions}

The EKF based SLAM approach of robot is investigated in this paper. When the noise model is unknown, the robustness 

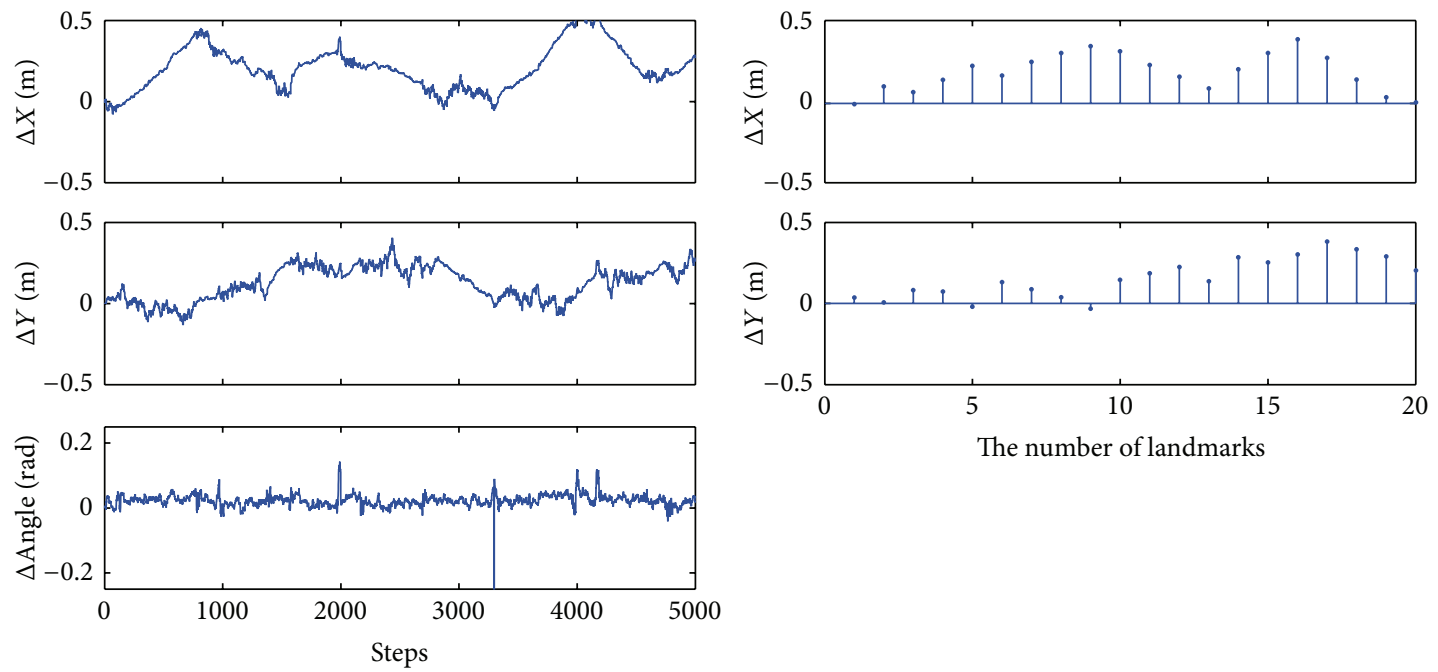

(a)
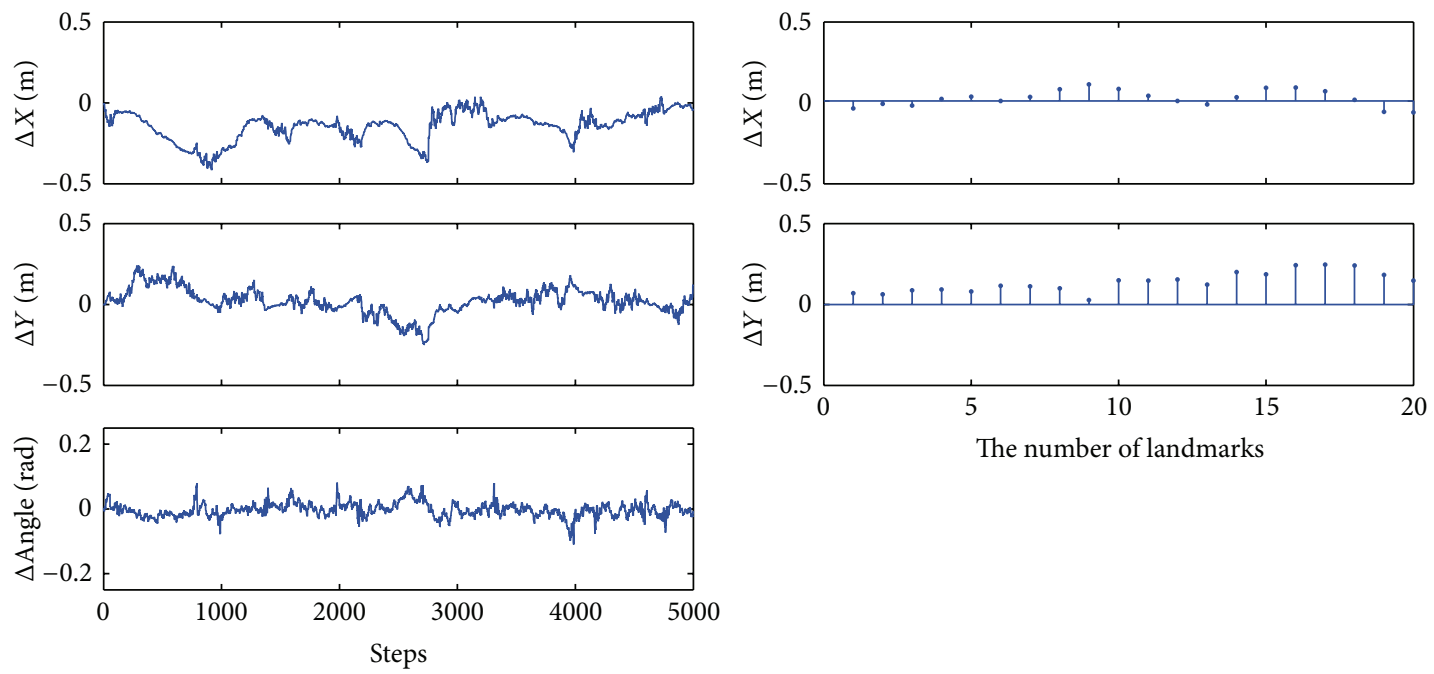

(b)
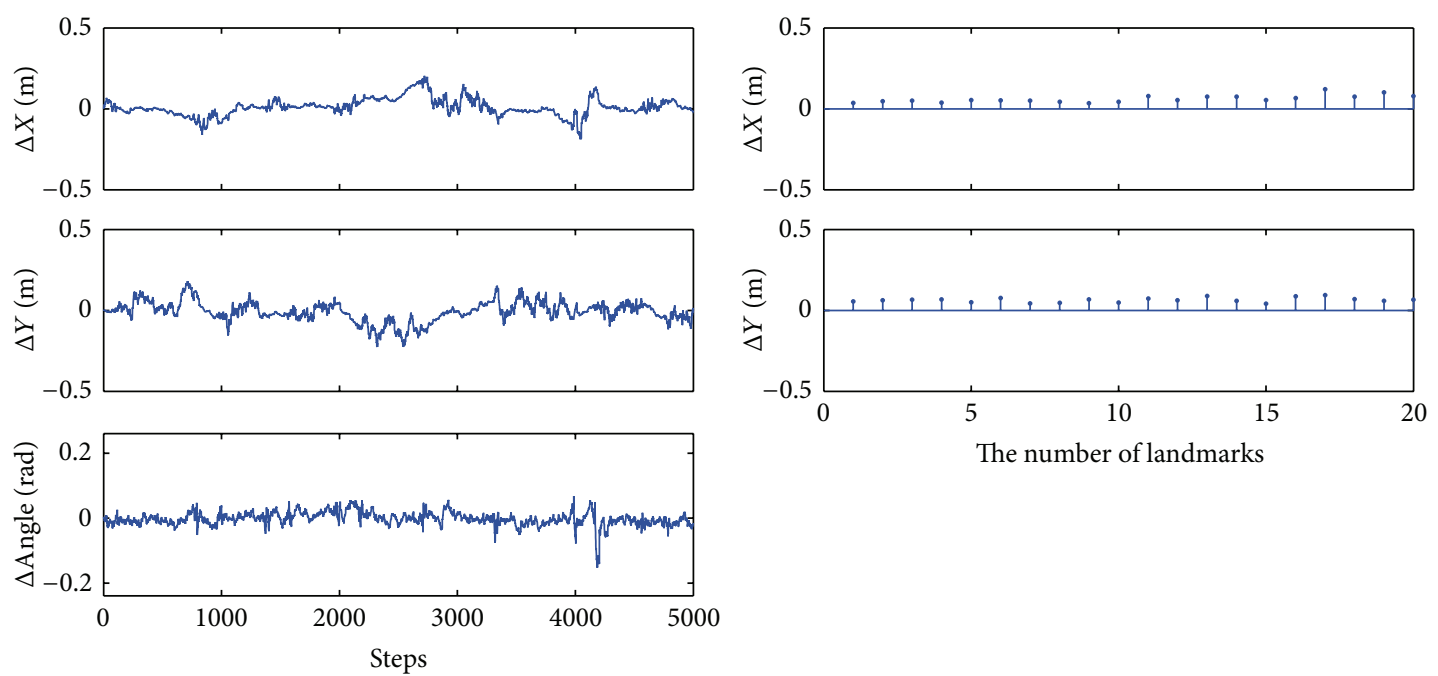

(c)

FIGURE 8: The localization error and the estimated error in the second experiment: (a) based on S-EKF method; (b) based on F-EKF method; (c) based on B-EKF method. 


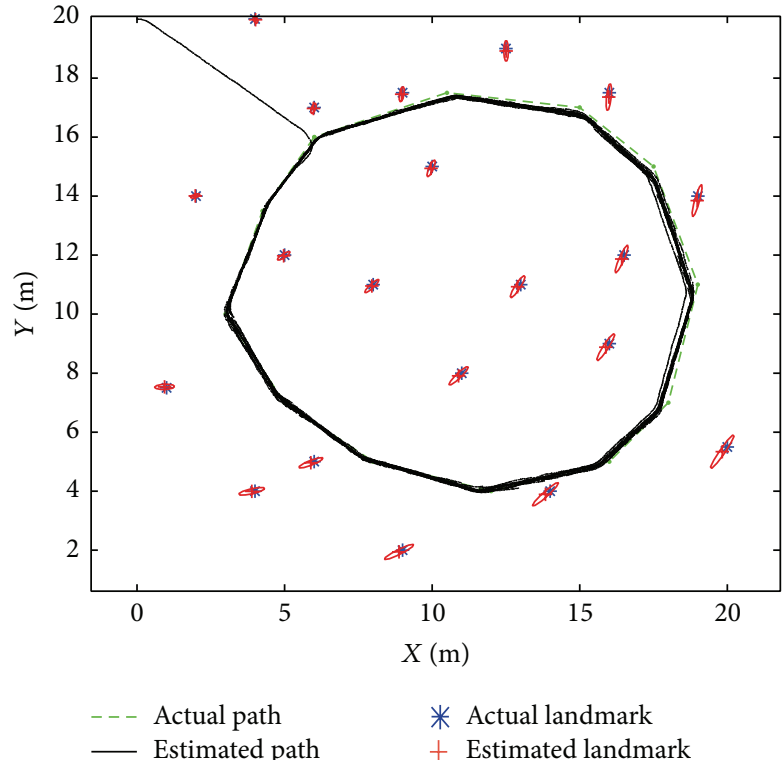

(a)

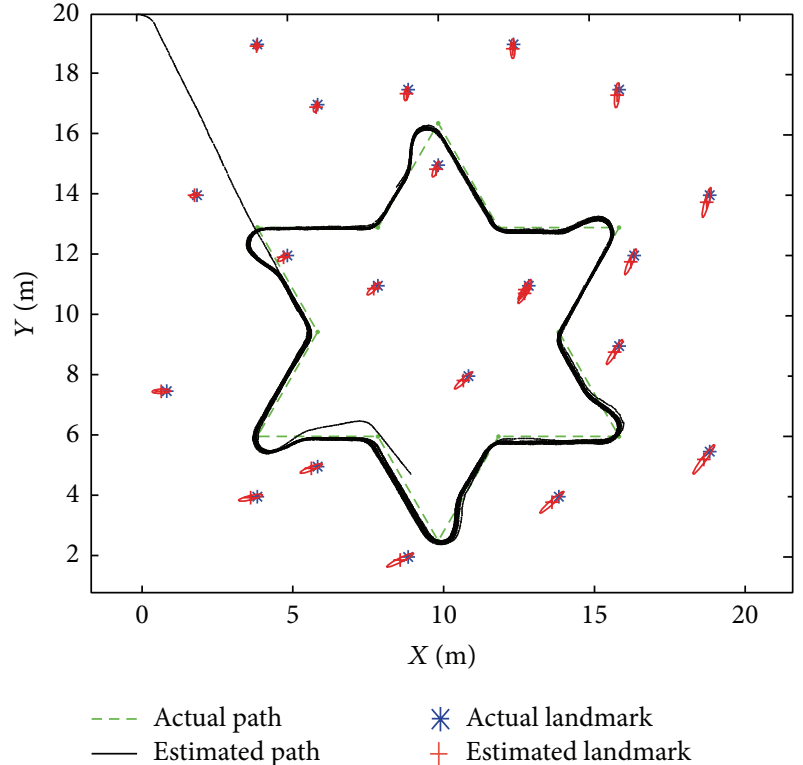

(b)

FIGURE 9: The results of the SLAM task based on the proposed approach with different trajectory and landmark distribution: (a) with a simple trajectory; (b) with a complex trajectory.

and accuracy of the EKF based SLAM algorithm will reduce. A novel adaptive EKF based SLAM algorithm structure is therefore proposed to adjust the weights of noises in the EKF based SLAM algorithm adaptively. Furthermore, a bioinspired neural model is integrated into this EKF based SLAM algorithm, which can reduce the error of the EKF based SLAM algorithm. As our approach is based on a bioinspired neural model, the SLAM task can be achieved efficiently, without any a priori knowledge of the noise model, nor any learning procedures. The feasibility and efficiency of the proposed approach have been discussed and illustrated through simulation studies. The proposed approach can deal with the searching and exploring problem in unknown environments, which has broad applications, such as the perceiving and understanding for the underwater and exoplanetary exploration.

In our research, there still remain some problems to be addressed. The first arises when the robot works in real and more complex environments. The second problem is the theoretical analysis of the bioinspired neural model based EKF for robot SLAM. In future work, some new bioinspired learning method and the $3 \mathrm{D}$ vision based method will be studied in the SLAM algorithm for robot.

\section{Conflict of Interests}

The authors declared that they have no conflict of interests to this work.

\section{Acknowledgments}

This work was supported by the National Natural Science Foundation of China (61203365), the Jiangsu Province
Natural Science Foundation (BK2012149), the Open Fund of Changzhou Key Laboratory of Sensor Networks and Environmental Sensing (CZSN201102), the Fundamental Research Funds for the Central Universities (2011B04614), and the Science and Technology Commission of Shanghai Municipality (12595810200).

\section{References}

[1] M. W. M. Gamini Dissanayake, P. Newman, S. Clark, H. F. Durrant-Whyte, and M. Csorba, "A solution to the simultaneous localization and map building (SLAM) problem," IEEE Transactions on Robotics and Automation, vol. 17, no. 3, pp. 229-241, 2001.

[2] K. Çelik and A. K. Somani, "Monocular vision SLAM for indoor aerial vehicles," Journal of Electrical and Computer Engineering, vol. 2013, Article ID 374165, 15 pages, 2013.

[3] D. Ribas, P. Ridao, J. D. Tardós, and J. Neira, "Underwater SLAM in man-made structured environments," Journal of Field Robotics, vol. 25, no. 11-12, pp. 898-921, 2008.

[4] Z. Li, Z. Xie, and A. Ming, "Simultaneously firing sonar ring based high-speed navigation for non- holonomic mobile robots in unstructured environment," International Journal of Vehicle Autonomous Systems, vol. 6, no. 1, pp. 172-185, 2008.

[5] S. Lee, S. Lee, and S. Baek, "Vision-based kidnap recovery with SLAM for home cleaning robots," Journal of Intelligent and Robotic Systems: Theory and Applications, vol. 67, no. 1, pp. 724, 2012.

[6] J. Mullane, B. Vo, M. D. Adams, and B. Vo, "A random-finiteset approach to Bayesian SLAM," IEEE Transactions on Robotics, vol. 27, no. 2, pp. 268-282, 2011.

[7] W. L. D. Lui and R. Jarvis, "A pure vision-based topological SLAM system," International Journal of Robotics Research, vol. 31, no. 4, pp. 403-428, 2012. 
[8] A. Chatterjee and F. Matsuno, "A neuro-fuzzy assisted extended kalman filter-based approach for simultaneous localization and mapping (SLAM) problems," IEEE Transactions on Fuzzy Systems, vol. 15, no. 5, pp. 984-997, 2007.

[9] W. Zhou, C. Zhao, and H. Zhang, "An AMPF and FastSLAM based compositive SLAM algorithm," Pattern Recognition and Artificial Intelligence, vol. 22, no. 5, pp. 718-725, 2009.

[10] M. Kaess, A. Ranganathan, and F. Dellaert, "iSAM: incremental smoothing and mapping," IEEE Transactions on Robotics, vol. 24, no. 6, pp. 1365-1378, 2008.

[11] D. Benedettelli, A. Garulli, and A. Giannitrapani, "Cooperative SLAM using M-Space representation of linear features," Robotics and Autonomous Systems, vol. 60, no. 10, pp. 1267-1278, 2012.

[12] J. Park, S. Lee, and J. Park, "Correction robot pose for SLAM based on extended Kalman filter in a rough surface environment," International Journal of Advanced Robotic Systems, vol. 6, no. 2, pp. 67-72, 2009.

[13] J. E. Guivant and E. M. Nebot, "Solving computational and memory requirements of feature-based simultaneous localization and mapping algorithms," IEEE Transactions on Robotics and Automation, vol. 19, no. 4, pp. 749-755, 2003.

[14] A. Gil, Ó. Reinoso, M. Ballesta, and M. Juliá, "Multi-robot visual SLAM using a Rao-Blackwellized particle filter," Robotics and Autonomous Systems, vol. 58, no. 1, pp. 68-80, 2010.

[15] S. Barkby, S. B. Williams, O. Pizarro, and M. V. Jakuba, "Bathymetric particle filter SLAM using trajectory maps," International Journal of Robotics Research, vol. 31, no. 12, pp. 1409-1430, 2012.

[16] J.-L. Blanco, J. González, and J.-A. Fernández-Madrigal, “Optimal filtering for non-parametric observation models: applications to localization and SLAM," International Journal of Robotics Research, vol. 29, no. 14, pp. 1726-1742, 2010.

[17] F. A. A. Cheein, F. M. L. Pereira, F. Di Sciascio, and R. Carelli, "Autonomous simultaneous localization and mapping driven by Monte Carlo uncertainty maps-based navigation," Knowledge Engineering Review, vol. 28, no. 1, pp. 35-57, 2013.

[18] M. Milford and G. Wyeth, "Persistent navigation and mapping using a biologically inspired SLAM system," International Journal of Robotics Research, vol. 29, no. 9, pp. 1131-1153, 2010.

[19] G. Wyeth and M. Milford, "Spatial cognition for robots: robot navigation from biological inspiration," IEEE Robotics and Automation Magazine, vol. 16, no. 3, pp. 24-32, 2009.

[20] A. Weitzenfeld, J.-M. Fellous, A. Barrera, and G. Tejera, "Allothetic and idiothetic sensor fusion in rat-inspired robot localization," in Multisensor, Multisource Information Fusion: Architectures, Algorithms, and Applications, vol. 8407 of Proceedings of SPIE, 2012.

[21] J. E. Guivant and E. M. Nebot, "Optimization of the simultaneous localization and map-building algorithm for real-time implementation," IEEE Transactions on Robotics and Automation, vol. 17, no. 3, pp. 242-257, 2001.

[22] H. Temeltas and D. Kayak, "SLAM for robot navigation," IEEE Aerospace and Electronic Systems Magazine, vol. 23, no. 12, pp. 16-19, 2008.

[23] L. Pedraza, D. Rodriguez-Losada, F. Matía, G. Dissanayake, and J. V. Miró, "Extending the limits of feature-based SLAM with Bsplines," IEEE Transactions on Robotics, vol. 25, no. 2, pp. 353366, 2009.

[24] L. de Souza Rosa and V. Bonato, "A method to convert floating to fixed-point EKF-SLAM for embedded robotics," Journal of the Brazilian Computer Society, vol. 19, no. 2, pp. 181-192, 2013.
[25] G. Sibley, C. Mei, I. Reid, and P. Newman, "Vast-scale outdoor navigation using adaptive relative bundle adjustment," The International Journal of Robotics Research, vol. 29, no. 8, pp. 958980, 2010.

[26] P. Loncomilla and J. R. Del Solar, "Visual SLAM based on rigidbody 3D landmarks," Journal of Intelligent and Robotic Systems: Theory and Applications, vol. 66, no. 1-2, pp. 125-149, 2012.

[27] S. Lei and Z. Li, "Fusing visual tracking and navigation for autonomous control of an intelligen t wheelchair," in Proceedings of the 3rd IFAC International Conference on Intelligent Control and Automation Science, pp. 549-554, Chengdu, China, 2013.

[28] S. Tully, G. Kantor, and H. Choset, "A unified Bayesian framework for global localization and SLAM in hybrid metric/topological maps," The International Journal of Robotics Research, vol. 31, no. 3, pp. 271-288, 2012.

[29] F.-M. Eduardo, G.-J. Javier, and A. Vicente, "Creating metrictopological maps for large-scale monocular SLAM," in Proceedings of the 10th International Conference on Informatics in Control, Automation and Robotics (ICINCO '13), vol. 2, pp. 3947, Reykjavik, Iceland, 2013.

[30] H. Du, Y. Hao, and Y. Zhao, "SLAM algorithm based on fuzzy adaptive Kalman filter," Journal of Huazhong University of Science and Technology (Natural Science Edition), vol. 40, no. 1, pp. 58-62, 2012.

[31] J. Kang, S. An, and S. Oh, "Modified neural network aided EKF based SLAM for improving an accuracy of the feature map," in Proceeding of the International Joint Conference on Neural Networks (IJCNN '10), pp. 1-7, Barcelona, Spain, July 2010.

[32] M. J. Milford, Robot Navigation from Nature: Simultaneous Localisation, Mapping, and Path Planning Based on Hippocampal Models, Springer, 2008.

[33] M. Pfingsthorn and A. Birk, "Simultaneous localization and mapping with multimodal probability distributions," International Journal of Robotics Research, vol. 32, no. 2, pp. 143-171, 2013.

[34] H. Choi, D. Y. Kim, J. P. Hwang, C.-W. Park, and E. Kim, "Efficient simultaneous localization and mapping based on ceilingview: ceiling boundary feature map approach," Advanced Robotics, vol. 26, no. 5-6, pp. 653-671, 2012.

[35] Y. Li, S. Li, and Y. Ge, "A biologically inspired solution to simultaneous localization and consistent mapping in dynamic environments," Neurocomputing, vol. 104, pp. 170-179, 2013.

[36] S. Huang and G. Dissanayake, "Convergence and consistency analysis for extended Kalman filter based SLAM," IEEE Transactions on Robotics, vol. 23, no. 5, pp. 1036-1049, 2007.

[37] H.-P. Li, D.-M. Xu, F.-B. Zhang, and Y. Yao, "Consistency analysis of EKF-based SLAM by measurement noise and observation times," Acta Automatica Sinica, vol. 35, no. 9, pp. 1177-1184, 2009.

[38] X. Ji, Z. Zheng, and H. Zhang, "Analysis and control of robot position error in SLAM," Acta Automatica Sinica, vol. 34, no. 3, pp. 323-330, 2008.

[39] S. X. Yang and M. Q.-. Meng, "Real-time collision-free motion planning of a mobile robot using a neural dynamics-based approach," IEEE Transactions on Neural Networks, vol. 14, no. 6, pp. 1541-1552, 2003.

[40] A. L. Hodgkin and A. F. Huxley, "A quantitative description of membrane current and its application to conduction and excitation in nerve," The Journal of Physiology, vol. 117, no. 4, pp. 500-544, 1952. 
[41] J. Ni and S. X. Yang, "Bioinspired neural network for realtime cooperative hunting by multirobots in unknown environments," IEEE Transactions on Neural Networks, vol. 22, no. 12, pp. 2062-2077, 2011.

[42] H. Qu, S. X. Yang, A. R. Willms, and Z. Yi, "Real-time robot path planning based on a modified pulse-coupled neural network model," IEEE Transactions on Neural Networks, vol. 20, no. 11, pp. 1724-1739, 2009.

[43] C. Luo and S. X. Yang, "A bioinspired neural network for realtime concurrent map building and complete coverage robot navigation in unknown environments," IEEE Transactions on Neural Networks, vol. 19, no. 7, pp. 1279-1298, 2008.

[44] D. Zhu, Y. Zhao, and M. Yan, "A bio-inspired neurodynamics based back stepping path-following control of an AUV with ocean current," International Journal of Robotics and Automation, vol. 27, no. 3, pp. 298-307, 2012.

[45] X. Yang, Neural network approaches to real-time motion planning and control of robotic systems [Ph.D. dissertation], Department of Electrical and Computer Engineering, University of Alberta, 1999.

[46] J. Kang, S. Kim, S. An, and S. Oh, "A new approach to simultaneous localization and map building with implicit model learning using neuro evolutionary optimization," Applied Intelligence, vol. 36, no. 1, pp. 242-269, 2012. 


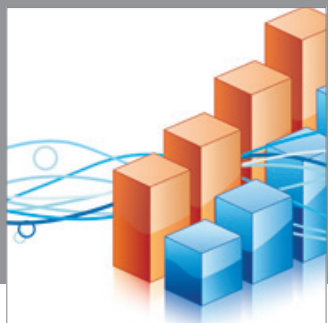

Advances in

Operations Research

mansans

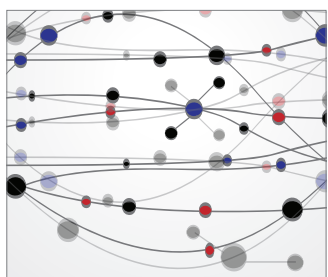

The Scientific World Journal
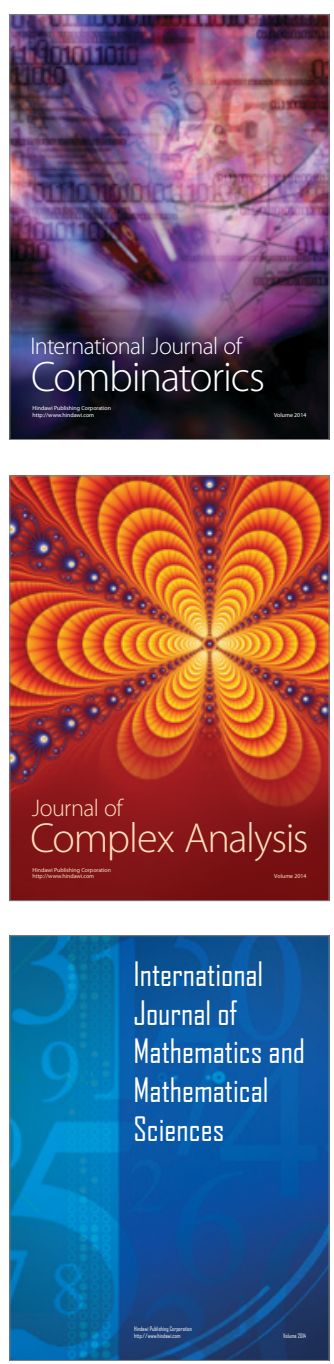
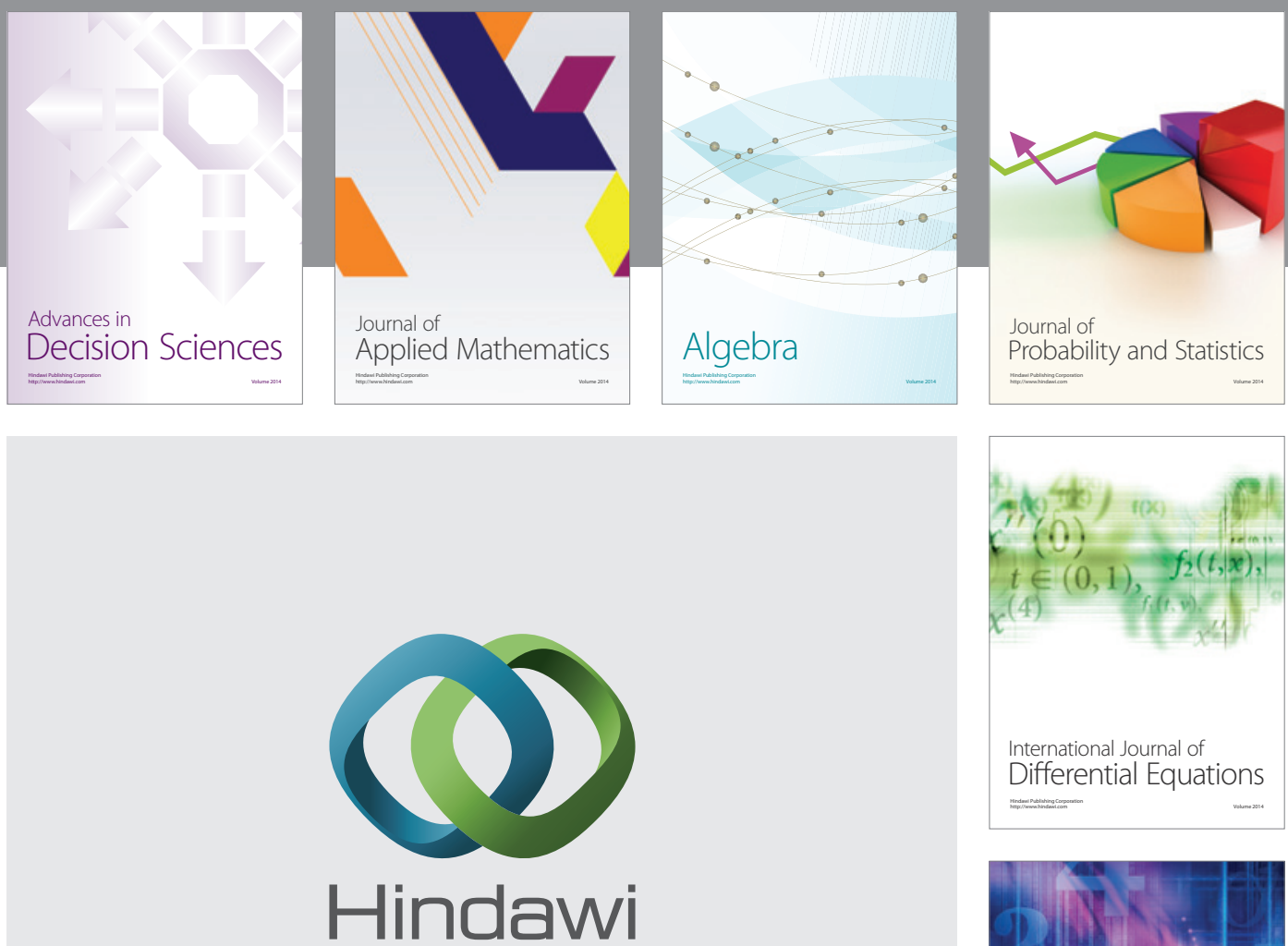

Submit your manuscripts at http://www.hindawi.com
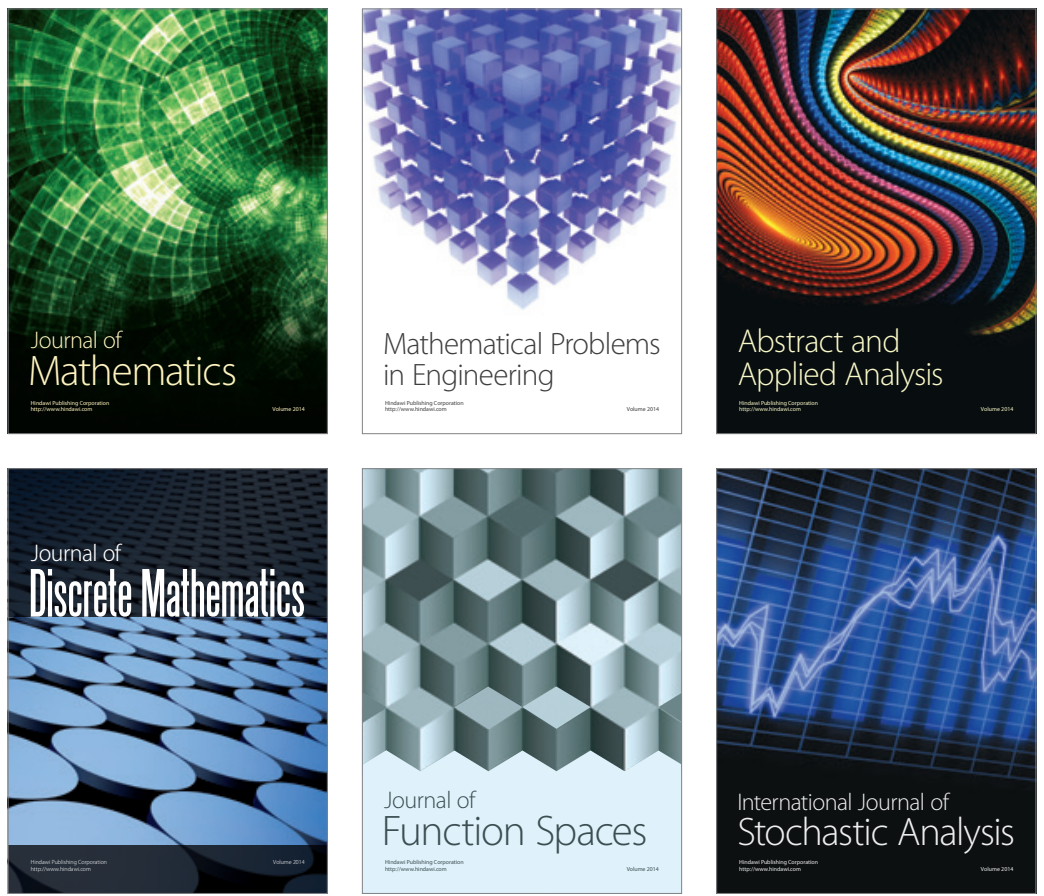

Journal of

Function Spaces

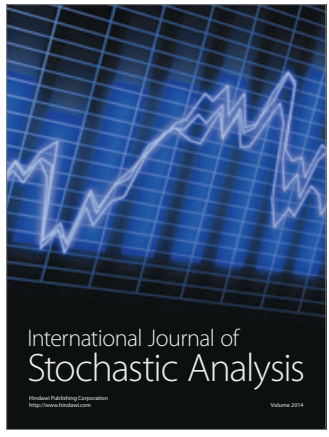

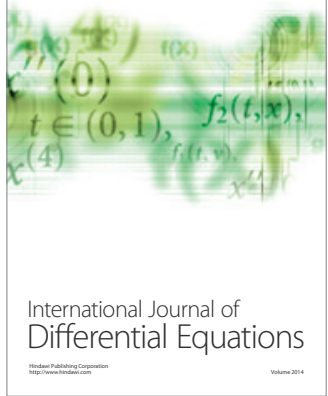
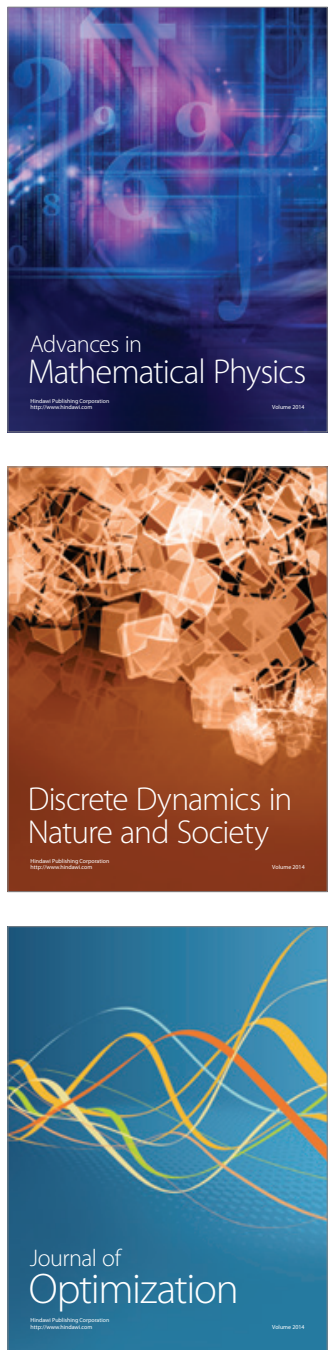